\title{
Gluon excitations of the static quark potential and the hybrid quarkonium spectrum*
}

\author{
K.J. Juge, J. Kuti, and C.J. Morningstar ${ }^{\mathrm{a}}$

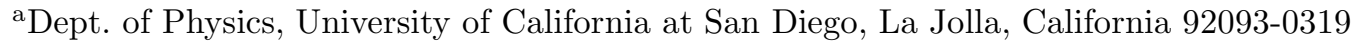

A comprehensive determination of the rich, low-lying spectrum of gluonic excitations in the presence of a static quark-antiquark pair is presented. Our results are obtained from several simulations on anisotropic lattices using an improved action and a large set of gluonic operators. The hybrid quarkonium states are studied in the quenched approximation using the Born-Oppenheimer expansion and nonrelativistic lattice QCD.

\section{Introduction}

Besides conventional hadrons, QCD suggests the existence of states containing gluonic excitations, such as glueballs and hybrid hadrons. Although conventional hadrons are reasonably well described by the constituent quark model, states with excited glue are still poorly understood. As experiments begin to focus on the search for glueballs and hybrid mesons, a better understanding of these states from theory is needed. Due to the highly nonperturbative nature of the gluonic excitations in these states, lattice simulations offer at present the best means of theoretically probing glueballs and hybrid mesons.

A great advantage in studying hybrid mesons comprised of heavy quarks is that such systems can be studied not only by direct numerical simulation, but also using the Born-Oppenheimer (BO) expansion. In this approach, the hybrid meson is treated analogous to a diatomic molecule: the slow heavy quarks correspond to the nuclei and the fast gluon field corresponds to the electrons $[1]$. First, one treats the quark $Q$ and antiquark $\bar{Q}$ as spatially-fixed colour sources and determines the energy levels of the glue as a function of the $Q \bar{Q}$ separation $r$; each of these energy levels defines a potential $V_{Q \bar{Q}}(r)$ adiabatically. The quark motion is then restored by solving the Schrödinger equation in each of these static potentials. Conventional quarkonia arise from

\footnotetext{
*Talk presented by C. Morningstar and poster presented
} by K.J. Juge. the lowest-lying static potential; hybrid quarkonium states emerge from the excited potentials. Once the static potentials have been determined (via lattice simulations), it is a simple matter to determine the complete conventional and hybrid quarkonium spectrum in the leading BornOppenheimer (LBO) approximation. This is a distinct advantage over meson simulations which yield only the very lowest-lying states, often with large statistical uncertainties.

Here, we present results for the spectrum of gluonic excitations in the presence of a static quarkantiquark pair. Some of these potentials have been studied before[2]. This study is the first to comprehensively survey the spectrum in $\mathrm{SU}(3)$ gauge theory. Due to our use of anisotropic lattices and an improved action, we have been able to determine the static potentials for much larger values of $Q \bar{Q}$ separation than previously studied. Using our potentials, we also determine the hybrid quarkonium spectrum. Results from a preliminary nonrelativistic lattice QCD simulation are also presented.

\section{Computation of the potentials}

The first step in the Born-Oppenheimer expansion is the determination of the rich spectrum of energy levels of the gluons in the presence of the quark and antiquark, fixed in space some distance $r$ apart. At this point in the approximation, the quark and antiquark simply act as static colour sources. The gluonic energies (or static potentials) may be labelled by the mag- 
Table 1

Simulation parameters, including the coupling $\beta$, input aspect ratio $\xi$, approximate lattice spacing $a_{s}$, lattice size, and spatial link smearing parameters ( $\lambda$ and $n_{\lambda}$ are defined in Ref. [3]).

\begin{tabular}{ccccc}
\hline$\beta$ & $\xi$ & $a_{s}(\mathrm{fm})$ & Lattice & $\left(\lambda, n_{\lambda}\right)$ \\
\hline 2.2 & 5 & 0.27 & $12^{3} \times 48$ & $(0.10,4)$ \\
& & & & $(0.20,4)$ \\
& & & & $(0.30,4)$ \\
\hline 2.4 & 5 & 0.23 & $14^{3} \times 56$ & $(0.10,8)$ \\
& & & & $(0.15,8)$ \\
& & & & $(0.20,8)$ \\
& & & & $(0.25,8)$ \\
\hline 2.6 & 3 & 0.19 & $10^{3} \times 30$ & $(0.15,8)$ \\
& & & & $(0.30,8)$ \\
\hline
\end{tabular}

nitude (denoted by $\Lambda$ ) of the projection of the total angular momentum of the gluons onto the molecular axis, by the sign of this projection (chirality or handedness), and by the behaviour under the combined operations of charge conjugation and spatial inversion about the midpoint between the quark and the antiquark. States with $\Lambda=0,1,2, \ldots$ are typically denoted by the capital Greek letters $\Sigma, \Pi, \Delta, \ldots$, respectively. States which are even (odd) under the above-mentioned parity-charge-conjugation operation are denoted by the subscripts $g(u)$. The energy of the gluons is unaffected by reflections in a plane containing the molecular axis; since such a reflection interchanges states of opposite handedness, such states must necessarily be degenerate ( $\Lambda$ doubling). However, this doubling does not apply to the $\Sigma$ states; $\Sigma$ states which are even (odd) under a reflection in a plane containing the molecular axis are denoted by a superscript $+(-)$. Hence, the low-lying levels are labelled $\Sigma_{g}^{+}, \Sigma_{g}^{-}, \Sigma_{u}^{+}, \Sigma_{u}^{-}$, $\Pi_{g}, \Pi_{u}, \Delta_{g}, \Delta_{u}$, and so on. For convenience, we use $\Gamma$ to denote these labels in general.

Static potentials were extracted from Monte Carlo estimates of generalized Wilson loops. On a starting time slice $t_{0}$, the quark and antiquark were fixed at lattice sites a distance $r$ apart. Several paths along the links of the lattice connecting the quark and the antiquark were then chosen, and our gluonic operators $O_{i}^{\Gamma}\left(t_{0}\right)$ were de-

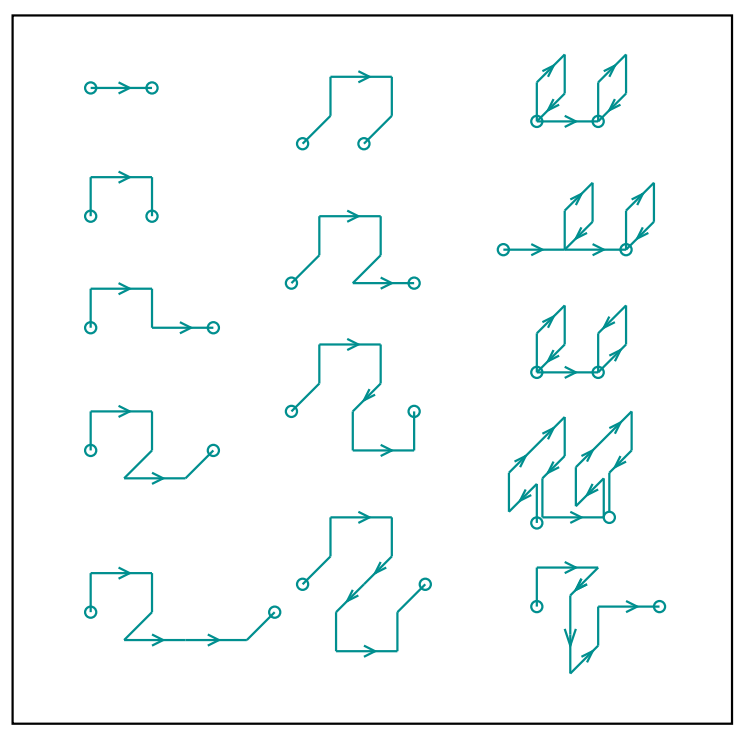

Figure 1. Examples of the paths from the quark to the antiquark used to construct the gauge field operators.

fined as linear combinations of the path-ordered exponentials of the gauge field along these paths. Examples of these paths are shown in Fig. 1. The linear combinations were chosen such that the operators transformed irreducibly under all symmetry operations. To reduce the mixings of our operators with excited states, the gluonic operators were constructed from iteratively-smeared spatial links. We used the single-link smearing algorithm described in Ref. [3]. The quark and antiquark then evolved in time, remaining fixed at their original spatial locations. To reduce statistical noise, the static quark propagators, which are simply temporal Wilson lines, were constructed from thermally-averaged temporal links [4], whenever possible. The thermal averaging was done using the pseudo-heat-bath method (40 updates). At some final time slice $t_{0}+\tau$, evaluation of the gluonic operators $O_{i}^{\Gamma \dagger}\left(t_{0}+\tau\right)$ then completed the construction of the Wilson loops $W_{i j}^{\Gamma}(r, \tau)$.

Monte Carlo estimates of the correlation matrices $W_{i j}^{\Gamma}(r, \tau)$ were obtained in three simulations. In each simulation, several spatial link smearing schemes were used: one scheme was typically cho- 


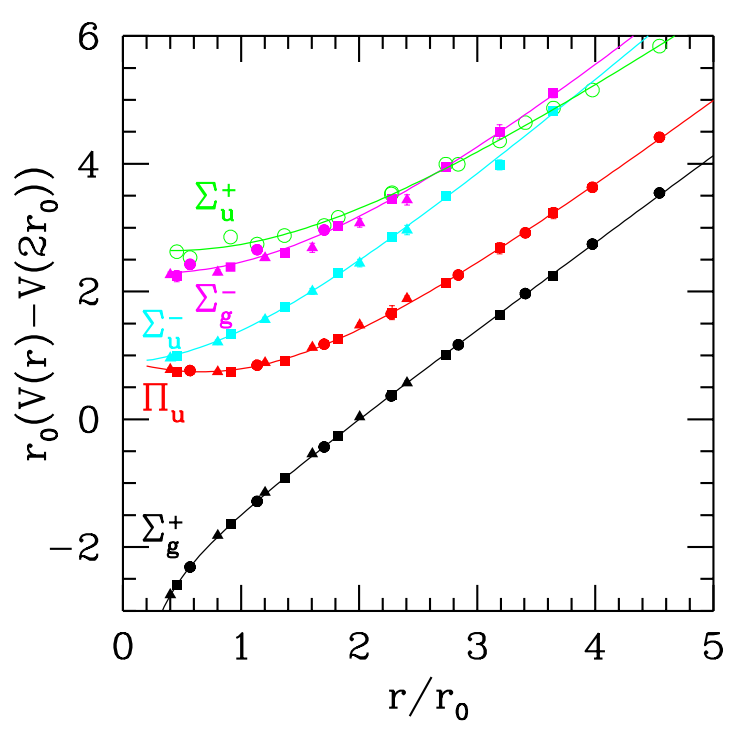

Figure 2. The static quark potential $V_{\Sigma_{g}^{+}}(r)$ and some of its gluonic excitations in terms of the hadronic scale parameter $r_{0}$ against the quarkantiquark separation $r$.

sen to work well for small $r$, another was optimized for large $r$. Various run parameters for the three simulations are given in Table 1; other relevant parameters can be found in Ref. 3]. We used the improved action described in Ref. [3]. Our use of anisotropic lattices in which the temporal lattice spacing $a_{t}$ was much smaller than the spatial spacing $a_{s}$ was crucial. Configuration ensembles were generated using a mixture of Cabibbo-Marinari (CM) pseudo-heat-bath and $\mathrm{SU}(2)$ subgroup over-relaxation (OR) methods.

The matrices $W_{i j}^{\Gamma}(r, \tau)$ were reduced in the data fitting phase to single correlators and $2 \times 2$ correlation matrices using the variational method described in Ref. [3]. These reduced correlators were fit using a single exponential and a sum of two exponentials in various ranges $t_{\min }$ to $t_{\max }$ of the source-sink separation. The two-exponential fits were used to check for consistency with the singleexponential fits, and in cases of favourable statistics, to extract the first-excited state energy. Best fit values were obtained using the correlated $\chi^{2}$ method. Error estimates were calculated using a 1024-point bootstrap procedure.

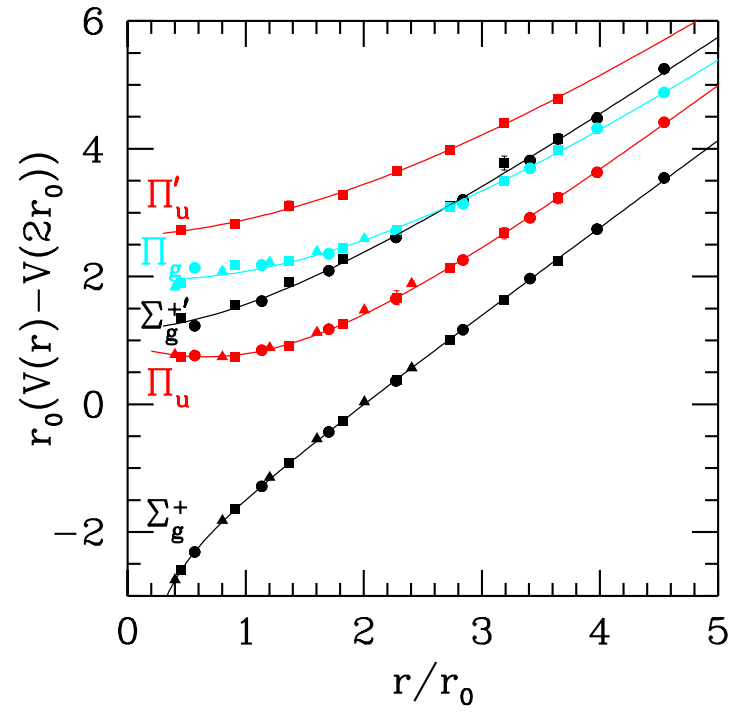

Figure 3. The static quark potential $V_{\Sigma_{g}^{+}}(r)$ and selected gluonic excitations (see Fig. 2).

Our results for the static potential and its gluonic excitations are shown in Figs. 2国. Results from the $\beta=2.2, \beta=2.4$, and $\beta=2.6$ runs are shown using solid circles, squares, and triangles, respectively. The results are expressed in terms of the hadronic scale parameter $r_{0}$. The definition of this parameter and a description of its calculation are given in Ref. [3]. The familiar static potential is shown as $\Sigma_{g}^{+}$; the solid curve is a fit to the data using a Coulomb plus linear form $V_{0}+e_{c} / r+\kappa r$. The curves for all other potentials are fits using $c_{0}+\sqrt{b_{0}+b_{1} r+b_{2} r^{2}}$. For all $r$ studied, the first-excited potential is the $\Pi_{u}$; hence, the lowest lying hybrid mesons must emerge from this potential.

As $r$ becomes very large, the linearly rising $\Sigma_{g}^{+}$ potential suggests that the ground state of the glue may be modelled as a fluctuating tube or string of colour flux; in this picture, the gluonic excitations are expected to be phonon-like with energy gaps proportional to $1 / r$. However, it appears that for $r$ below about $1.5 \mathrm{fm}$, the gluonic spectrum cannot be explained in terms of a simple string model. In Ref. [1], a QCD motivated bag model was successfully used to describe both the $\Sigma_{g}^{+}$and $\Pi_{u}$ potentials for a large range of $r$. 


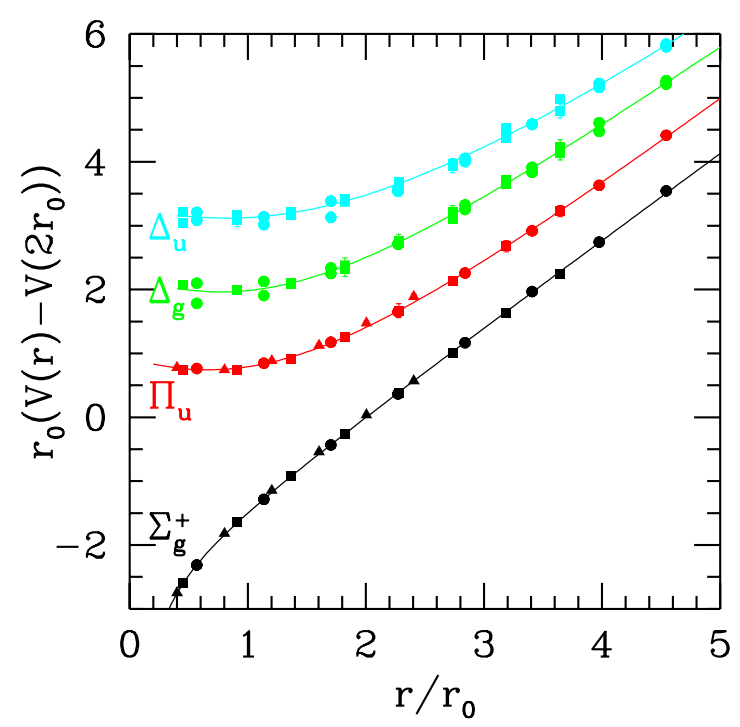

Figure 4. The static quark potential $V_{\Sigma_{g}^{+}}(r)$ and selected gluonic excitations (see Fig. 2).

In this picture, the strong chromoelectric fields of the quark and antiquark repel the physical vacuum (dual Meissner effect), creating a bubble inside which perturbation theory is applicable. In the ground state, the inward pressure on the bubble from the physical vacuum balances the outward chromostatic force in such a way to produce a linearly confining potential. The addition of one or more gluons into the bag produces the excited potentials; the kinetic energy of the gluons inside the bubble is a key factor in determining the form of these potentials. This model has recently been revisited and results (in the ellipsoidal approximation) for almost all of the potentials studied here are in remarkable agreement with our findings from the lattice simulations (see Ref. (50).

\section{Hybrid quarkonium}

The next step in the BO expansion is to restore the quark motion by solving the radial Schrödinger equation,

$\frac{d^{2} u(r)}{d r^{2}}+2 \mu\left[E-V_{\mathrm{eff}}(r)\right] u(r)=0$,

where $V_{\text {eff }}(r)=V_{Q \bar{Q}}(r)+\left\langle\mathbf{L}_{Q \bar{Q}}^{2}\right\rangle /\left(2 \mu r^{2}\right), \mu$ is the reduced mass, and $\varphi(r)=u(r) / r$ is the radial wavefunction. The total angular momentum of the meson is given by $\mathbf{J}=\mathbf{L}+\mathbf{S}$, where $\mathbf{S}$ is the sum of the spins of the quark and antiquark, and the orbital factor $\mathbf{L}=\mathbf{L}_{Q \bar{Q}}+\mathbf{J}_{g}$, where $\mathbf{J}_{g}$ is the total angular momentum of the glue and $\mathbf{L}_{Q \bar{Q}}$ is the orbital angular momentum of the quark and antiquark. In the LBO approximation, the eigenvalues $L(L+1)$ and $S(S+1)$ of $\mathbf{L}^{2}$ and $\mathbf{S}^{2}$ are good quantum numbers. The centrifugal factor is then written as

$\left\langle\mathbf{L}_{Q \bar{Q}}^{2}\right\rangle=L(L+1)-2 \Lambda^{2}+\left\langle\mathbf{J}_{g}^{2}\right\rangle$.

For the $\Sigma_{g}^{+}$potential, $\left\langle\mathbf{J}_{g}^{2}\right\rangle=0$. For the $\Pi_{u}$ and $\Sigma_{u}^{-}$potentials, we assumed that the one gluon state was dominant with $\left\langle\mathbf{J}_{g}^{2}\right\rangle=2$. Mesonic eigenstates of parity and charge-conjugation are linear combinations of left- and right-handed glue states: $\mid$ left $\rangle+\epsilon \mid$ right $\rangle$, where $\epsilon= \pm 1$. Let $\eta= \pm 1$ denote the $P C$ quantum number of the glue. Then in the LBO approximation, the parity $(\mathrm{P})$ and charge conjugation $(\mathrm{C})$ of each meson is given in terms of $L$ and $S$ according to

$P=\epsilon(-1)^{L+\Lambda+1}$,
$C=\epsilon \eta(-1)^{L+\Lambda+S}$.

The potentials computed from Wilson loops in lattice simulations contain the self-energies of the temporal Wilson lines. These self-energy contributions are common to all of the static potentials and must be removed in order to obtain the $V_{Q \bar{Q}}(r)$ appearing in Eq. 1. Ideally, this can be done by measuring the $\Sigma_{g}^{+}$potential for very small $r$ and comparing with the running Coulomb law as predicted from perturbation theory. In practice, this is difficult to do. Instead, we fit our results for the $\Sigma_{g}^{+}$potential to the form $V_{0}+e_{c} / r+\kappa r$; the constant $V_{0}$ is then our estimate of the self-energy contributions to be removed.

Results for the $b \bar{b}$ spectrum are shown in Fig. 5 . The scale was set using $r_{0}^{-1}=430 \mathrm{MeV}$ as suggested from NRQCD simulations of $b \bar{b}$ and $c \bar{c}$ mesons (see Table XX of Ref. [3]). The heavy quark mass $M_{b}$ was tuned in order to reproduce the experimentally-known $\Upsilon(1 S)$ mass: $M_{\Upsilon}=$ $2 M_{b}+E_{0}$, where $E_{0}$ is the energy of the lowestlying state in the $\Sigma_{g}^{+}$potential. In the LBO approximation, many mesons are degenerate: the 


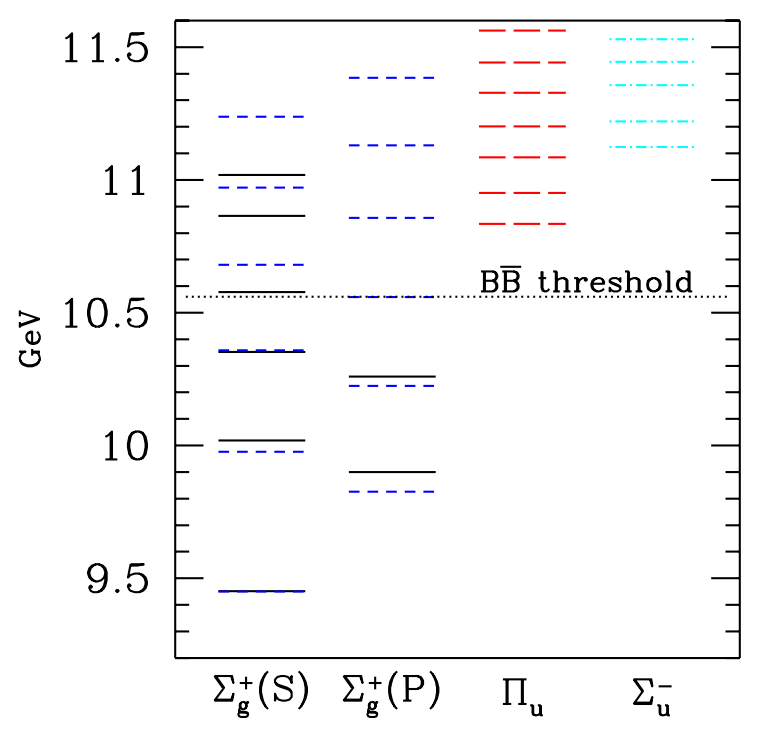

Figure 5. Spin-averaged $b \bar{b}$ spectrum in the leading Born-Oppenheimer and quenched approximations. Solid lines indicate experimental measurements. Short dashed lines indicate the $S$ and $P$ state masses obtained by solving the appropriate Schrödinger equation in the $\Sigma_{g}^{+}$potential using $r_{0}^{-1}=0.430 \mathrm{GeV}$ and $M_{b}=4.60 \mathrm{GeV}$ for the heavy quark mass. Long dashed and dashed-dotted lines indicate the hybrid quarkonium states obtained from the $\Pi_{u}(L=1,2)$ and $\Sigma_{u}^{-}(L=0,1,2)$ potentials, respectively.

$J^{P C}=0^{-+}, 1^{--} S$-waves from the $\Sigma_{g}^{+}$potential are degenerate; the $0^{++}, 1^{++}, 2^{++}, 1^{+^{-}} P$-waves from the $\Sigma_{g}^{+}$potential have equal masses; states such as $0^{-+}, 0^{+-}, 1^{++}, 1^{-+}, 1^{--}, 1^{+-}$from the $\Pi_{u}$ potential are also degenerate.

Below the $B \bar{B}$ threshold, the LBO results are in very good agreement with the spin-averaged experimental measurements. Note that these results make use of the quenched potentials (which ignore the light quarks) and do not include spin, retardation, and other relativistic effects. Above the threshold, agreement with experiment is lost, suggesting significant corrections from either the light quarks, relativistic effects, or possibly mixings between the states from the different adiabatic potentials. Note that the mass of the lowest-

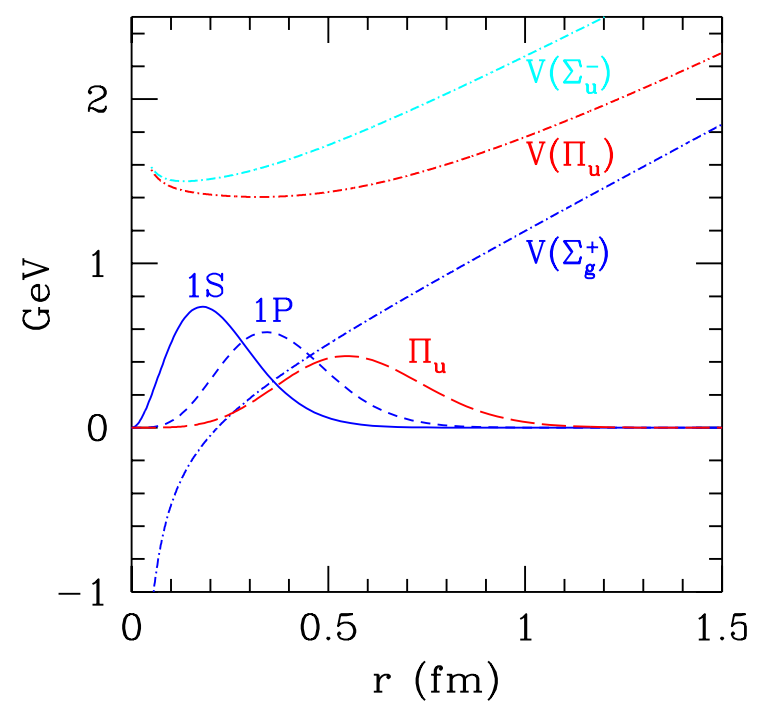

Figure 6. Static potentials and radial probability densities against quark-antiquark separation $r$. The $\Sigma_{g}^{+}$static potential and the $\Pi_{u}$ and $\Sigma_{u}^{-}$ excitations are indicated by the dashed-dotted lines. The solid and short-dashed curves indicate the radial probability densities for the $1 S$ and $1 P$ states, respectively, corresponding to the results shown in Fig. 5. The extended nature of the lowest-lying $\Pi_{u}$ hybrid state is shown by the radial probability density indicated by the longdashed curve.

lying hybrid (from the $\Pi_{u}$ potential) is about $10.8 \mathrm{GeV}$. Hybrid mesons from all other static potentials are significantly higher lying. Above $11 \mathrm{GeV}$, the LBO approximation based on the quenched $V_{Q \bar{Q}}$ potentials predicts a very dense population of hybrid states. The radial probability densities for the $1 S$ and $1 P$ conventional states are compared with that of the lowest-lying $\Pi_{u}$ hybrid state in Fig. 6.

\section{NRQCD simulations}

Hybrid quarkonium states may also be studied directly in numerical simulations. The nonrelativistic formulation of lattice QCD (NRQCD) [6] is a particularly efficient means of carrying out such simulations. We have recently begun an investigation of the hybrid quarkonium states using 


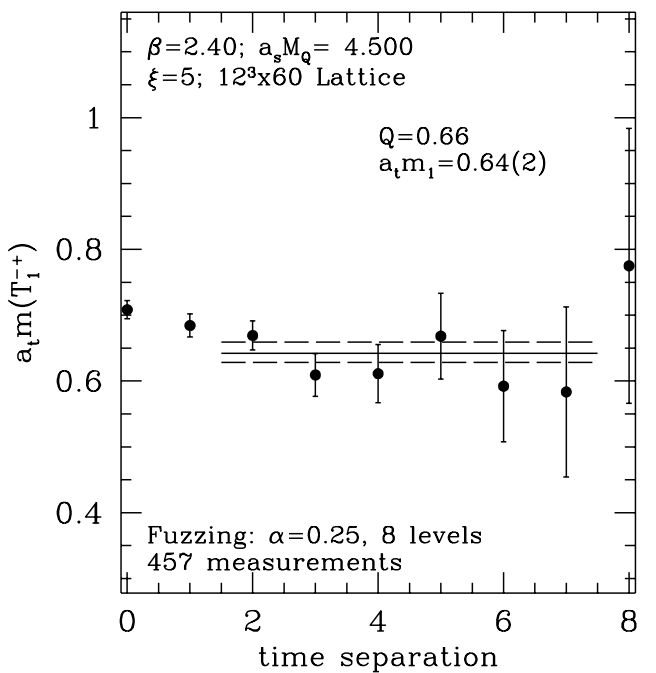

Figure 7. Effective mass plot showing the results of a single-exponential fit to the correlation function of the hybrid $T_{1}^{-+}$quarkonium state. The heavy quark propagates according to a spinindependent NRQCD action.

a spin-independent version of the lattice NRQCD action. The action included only a covariant temporal derivative and the leading kinetic energy operator (with two other operators to remove $O\left(a_{t}\right)$ and $O\left(a_{s}^{2}\right)$ errors); relativistic corrections depending on spin, $\mathbf{E}$ and $\mathbf{B}$, and higher derivatives were not included. The action was chosen to correspond as closely as possible to the LBO approximation. In so doing, our treatments of the hybrid mesons using the Born-Oppenheimer expansion and using NRQCD simulations differed in primarily two aspects: lattice spacing errors and retardation effects. The NRQCD simulations included retardation effects since the covariant Laplacian of the quark kinetic energy operator was treated exactly. In contrast, the LBO approximation ignored all retardation effects by keeping only the leading term in the $1 / c$ expansion of the covariant Laplacian. A comparison of results from the two approaches should afford a test of the adiabatic approximation.

Because the signal-to-noise ratio was expected to be poor and the masses of the hybrid mesons were expected to be large, it was crucial to use an anisotropic lattice in which the temporal lattice spacing was much smaller than the spatial spacing. The operators used to calculate the static potentials were incorporated into our NRQCD simulation code. We constructed large sets of gaugeinvariant operators in order to minimize excitedstate contamination of our hybrid meson correlators using variational techniques. We have completed an initial run on a $12^{3} \times 60$ lattice using an aspect ratio $\xi=5$ (that is, $a_{s}=5 a_{t}$ ). As the purpose of this run was merely to discover the quality of signal which was possible, the quark mass was not tuned and a value $a_{s} M_{b}=4.50$ was used. The effective mass from this run for the $1^{-+}$hybrid meson is shown in Fig. 7. A convincing plateau was observed from 457 measurements, and we were able to extract the mass with a $3 \%$ statistical uncertainty.

\section{Conclusion}

A first comprehensive survey of the spectrum of quenched $\mathrm{SU}(3)$ gluonic excitations in the presence of a static $Q \bar{Q}$ pair was presented. The hybrid quarkonium states were calculated in the leading Born-Oppenheimer approximation. The effective mass for the $1^{-+}$hybrid from a preliminary NRQCD simulation was also shown. This work was supported by the U.S. DOE, Grant No. DE-FG03-90ER40546.

\section{REFERENCES}

1. P. Hasenfratz, R. Horgan, J. Kuti, J. Richard, Phys. Lett. 95B, 299 (1980).

2. S. Perantonis and C. Michael, Nucl. Phys. B347, 854 (1990); I.J. Ford, R.H. Dalitz, and J. Hoek, Phys. Lett. B208, 286 (1988); N. Campbell et al., Phys. Lett. 142B, 291 (1984).

3. C. Morningstar and M. Peardon, Phys. Rev. D 56, 4043 (1997).

4. G. Parisi et al., Phys. Lett. 128B, 418 (1983).

5. K.J. Juge, J. Kuti, and C.J. Morningstar, these proceedings.

6. G. P. Lepage, L. Magnea, C. Nakhleh, U. Magnea, and K. Hornbostel, Phys. Rev. D 46, 4052 (1992). 